\title{
MICROSTRUCTURE AND CRYSTALLOGRAPHIC TEXTURE OF SILICON IRON MODIFIED BY TORSION UNDER QUASI- HYDROSTATIC PRESSURE
}

\author{
A. M. Glezer, ${ }^{1,2}$ A. N. Belyakov, ${ }^{3}$ G. R. Rostovtsev, \\ M. V. Odnobokova, ${ }^{3}$ I. V. Shchetinin, ${ }^{1}$ and A. A. Tomchuk ${ }^{2,4}$
}

UDC 539.25

The electron backscatter diffraction, X-ray diffraction analysis, electromotive force instantaneous measurement, microhardness and coercive force measurement techniques are used to explore the development of the microstructure, crystallographic texture and physico-mechanical properties of silicon iron (Fe-3\% $\mathrm{Si}$ ) alloy under quasi-hydrostatic pressure in a Bridgman anvil. It is found that the alloy deformation is accompanied by its significant hardening. In-plane torsion test shows that the average grain size rapidly decreases down to $185 \mathrm{~nm}$ after a quater turn of the movable anvil. With the number of turns increased up to six the grain size gradually decreases down to $150 \mathrm{~nm}$. At the same time, the average size of subgrains is less dependent on the deformation degree. The crystallographic texture evolution involves the formation of the strong $\{001\}<110>$ texture component after comparatively small megaplastic deformation followed by the simple shear texture formation. Such a behavior can be correctly described in terms of non-equilibrium thermodynamics.

Keywords: large deformation, structure, texture, coercive force, microhardness, non-equilibrium thermodynamics.

\section{INTRODUCTION}

Silicon iron (Fe-3 wt.\% $\mathrm{Si}$ ) is a model alloy composition which is used to study the plastic deformation behavior and the formation of crystallographic texture in metallic crystals with the body-centered cubic (BCC) crystal lattice [1]. This alloy is characterized by the absence of the high-temperature $\gamma \rightarrow \alpha$ phase transfer and is used to obtain grain-oriented electrical steel with low electromagnetic losses [2].

As is known, the production of ultra-fine grain (UFG) and nanocrystalline materials is efficient when utilizes large plastic deformation processing at relatively low temperatures. To the date, researchers worldwide have accumulated successful experimental experience in the production of metallic materials with the UFG and nanocrystalline structures using various methods of plastic deformation [3]. At the same time, the main structural changes responsible for the UFG and nanocrystalline structure formation driven by large plastic deformations merit a deep and systematic analysis.

The limitations of research in the field of microstructure and texture modifications caused by large plastic deformations arisen from complexities of getting reliable results. In particular, this concerns torsion under quasi-

${ }^{1}$ The National University of Science and Technology "MISiS”, Moscow, Russia, e-mail: ingvar@misis.ru; ${ }^{2}$ The Bardin Central Research Institute of Ferrous Metallurgy, Moscow, Russia, e-mail: a.glezer@mail.ru; rostovtcevgrisha@rambler.ru; ${ }^{3}$ National Research Belgorod State University, Belgorod, Russia, e-mail: belyakov@bsu.edu.ru; odnobokova@bsu.edu.ru; ${ }^{4}$ The Bauman Moscow State Technical University, Moscow, Russia, e-mail: tomchuk-a@yandex.ru. Translated from Izvestiya Vysshikh Uchebnykh Zavedenii, Fizika, No. 8, pp. 196-204, August, 2019. Original article submitted June 25, 2019. 
hydrostatic pressure in a Bridgman anvil used for studying plastic deformation [4]. In this case, the defect density of the crystalline structure sharply rises involving the dislocation substructure which significantly distorts the microstructure images obtained by conventional techniques of electron microscopy [5]. Moreover, a small size of samples also complicates the quantification analysis of the structure and crystallographic texture of the material. The development of the UFG structure is mainly studied on samples deformed at relatively high homologous temperatures. The up-to-date scanning electron microscopy combined with the electron backscatter diffraction (EBSD) technique which is used in the study of crystalline or polycrystalline materials allows us to fill the gap in the qualitative analyses of UFG and nanocrystalline metallic materials subjected to cold plastic deformation, including high-melting steel and alloy processing in a Bridgman anvil.

At the same time, very little published information is available concerning the behavior of silicon iron exposed to large plastic deformations [6]. This work aims to investigate the evolution of the microstructure, crystallographic texture and physico-mechanical properties of silicon iron during large (megaplastic) deformation and torsion under quasi-hydrostatic pressure in the Bridgman anvil. Special attention in this process is given to the analysis of changes in the crystallographic texture.

\section{MATERIALS AND METHODS}

Electrical steel ( $3.14 \mathrm{wt} . \% \mathrm{Si})$ sheets $0.35 \mathrm{~mm}$ thick were used in this experiment, which after the secondary recrystallization possessed the Goss texture of (110) [001] orientation and coarse grains (about 15-20 mm). The steel composition included a small amount of impurities (not over $0.1 \mathrm{wt} . \% \mathrm{C}, \mathrm{S}, \mathrm{N}, \mathrm{O}, \mathrm{Al}, \mathrm{Ni}$ and $\mathrm{Mn}$ ). This steel was used to fabricate discs $0.35 \mathrm{~mm}$ thick and $6 \mathrm{~mm}$ in diameter, which were annealed in argon at $800^{\circ} \mathrm{C}$ for 50 hours and then cooled down to room temperature at a cooling rate of 100 degrees per hour. Since the diameter of the obtained discs was less than the mean diameter of the grains in the initial state, the disc samples were positioned inside only one grain and had the single-crystal orientation approaching to (110) [001]. The disc samples of silicon iron underwent plastic deformation under quasi-hydrostatic pressure in the Bridgman anvil. The pressure conditions included room temperature $(295 \mathrm{~K}) ; n=1 / 4,1 / 2,1,2,3,4$ and 6 number of turns of the movable anvil; 6 GPa quasi-hydrostatic pressure; $1 \mathrm{rpm}$ rotating speed.

The true logarithmic strain $e$ under the quasi-hydrostatic pressure is obtained from [4]

$$
e=\ln \left(1+\left(\frac{\varphi \cdot r}{h}\right)^{2}\right)^{0.5}+\ln \left(\frac{h_{0}}{h}\right)
$$

where $r$ and $h$ are respectively the radius and the height of the sample, $\varphi$ is the angle of rotation. According to Eq. (1), the true logarithmic strain $e$ is varied within 5-9.

The structure investigations and measurements of the hardness and coercive force were carried out at halfradius in-plane shear. The microstructure and crystallographic texture were studied on a Nova NanoSEM 450 fieldemission scanning electron microscope (JEOL, Japan) equipped with a backscatter electron detector and an automatic analysis system for the crystal lattice orientation. The scanning step was $25 \mathrm{~nm}$. The orientation imaging microscopy (OIM), developed by TexSEM Laboratories Inc. utilized the OIM Analysis 6 software for the quantitative and qualitative analyses of the microstructure and crystallographic texture. In the obtained OIM maps of the crystal lattice orientations, the insufficient crystallographic points were replaced by the minimum grain size of 5 crystallographic points at a mutual misorientation angle of not over 5 degrees. The crystallographic point orientation with the confidence index less than 0.05 was not considered. Such crystallographic points were represented on OIM maps by black spots. The grain size was determined as a circle diameter of the equivalent area with the boundaries with a misorientation angle of 15 degrees and above. Subgrains on the grain boundary maps represented areas with the grain-boundary misorientation angle of not less than 2 degrees with regard to the space resolution used in the given methodology [7]. The X-ray diffraction (XRD) analysis was carried out on a Rigaku Ultima IV diffratometer before and after quasihydrostatic pressure, using $\mathrm{Cr} K_{\alpha}$ radiation and a graphite monochromator. The Rigaku Ultima IV worked in the Bragg- 


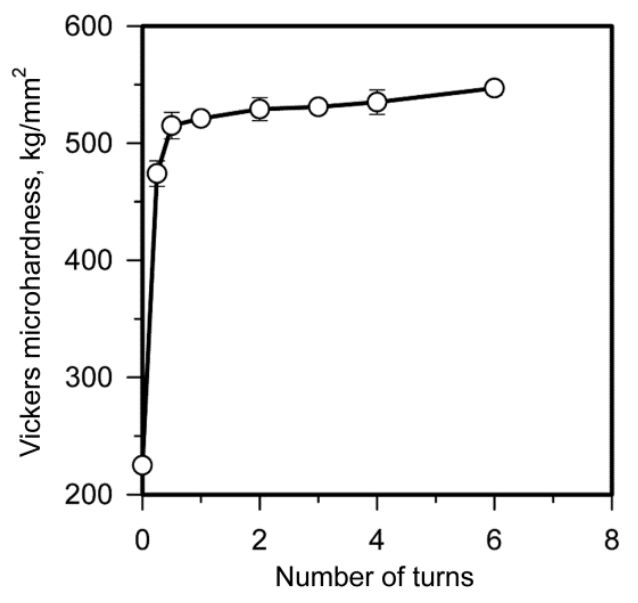

Fig. 1. Dependence between megaplastic deformation and microhardness of $\mathrm{Fe}-3 \%$ Si alloy sample.

Brentano geometry (30-125 degrees), at a $0.1^{\circ}$ scanning step. The modified Rietveld refinement method was used to analyze the XRD patterns. The BCC crystal lattice parameter of $\alpha$-phase and the lattice microdistortions were considered as structural parameters before and after the quasi-hydrostatic pressure applied. The integrated analysis was performed for the whole sample surface. The PDXL full-function powder diffraction analysis package (Rigaku Corp., Japan) [8] was used for data processing in view of the Debye-Waller and the Lorentz factors, and crystallographic texture. The measurement accuracy of the lattice constant was \pm 0.0005 A. A Rigaku SmartLab X-ray diffractometer was used for displaying the crystallographic texture via pole figures.

Coercive force measurements were conducted at room temperature. A VSM-250 vibrating-sample magnetometer was used to measure the magnetic properties. The DC magnetic field ranged from 80 to $640 \mathrm{kA} / \mathrm{m}$, with a minimum interval of $0.8 \mathrm{~A} / \mathrm{m}$. The VSM-250 was calibrated using the reference sample of pure nickel. We selected perfectly round test pieces without cracks. A LECO M400 Vickers hardness tester was used to determine the microhardness at a $50 \mathrm{~g}$ load for 5 seconds. The electromotive force (the difference between the Gibbs energy of deformed and non-deformed alloy samples) instantaneous measurement was carried out on the experimental setup designed in [9].

\section{RESULTS AND DISCUSSION}

According to Fig. 1, megaplastic deformation of silicon iron (Fe-3\% $\mathrm{Si}$ ) in the Bridgman anvil provides a significant hardening of the material. Vickers microhardness values (HV) sharply grow from $2250 \mathrm{MPa}$ in the initial state to $4740 \mathrm{MPa}$ after a quarter turn of the movable anvil. At the same time, the intensity of hardening notably lowers with increasing number of turns. After a half turn, the HV value grows up to $515 \mathrm{MPa}$, and further megaplastic deformation is accompanied by the increase in this value with almost constant strain-hardening index $n$. When the latter achieves 6, the $\mathrm{HV}$ value equals $5470 \mathrm{MPa}$.

In Fig. 2 one can see a qualitative correlation between the structural changes and changes in the HV value. After a quarter turn, the grain size of the appeared UFG structure is $185 \mathrm{~nm}$, the average grain size being $125 \mathrm{~nm}$. The rate of grain grinding gradually lowers in the course of megaplastic deformation. The highest grinding rate is observed at $n \leq 2$. After $n=2$, the grain size achieves $160 \mathrm{~nm}$. A further increase in the number of turns up to 6 causes a gradual decrease in the grain size down to $150 \mathrm{~nm}$. The decreased grain size mostly contributes to the sample hardening. The dependence of the grain and subgrain sizes $D$ and $d$ of $\mathrm{Fe}-3 \% \mathrm{Si}$ alloy sample on the quasi-hydrostatic pressure is presented in Fig. 3. Unlike the grain size, the size of subgrains is significantly less dependent on deformation. Subgrains sufficiently decrease in size (down to $115 \mathrm{~nm}$ ) only at $n<2$. The increased number of turns does not affect the subgrain 

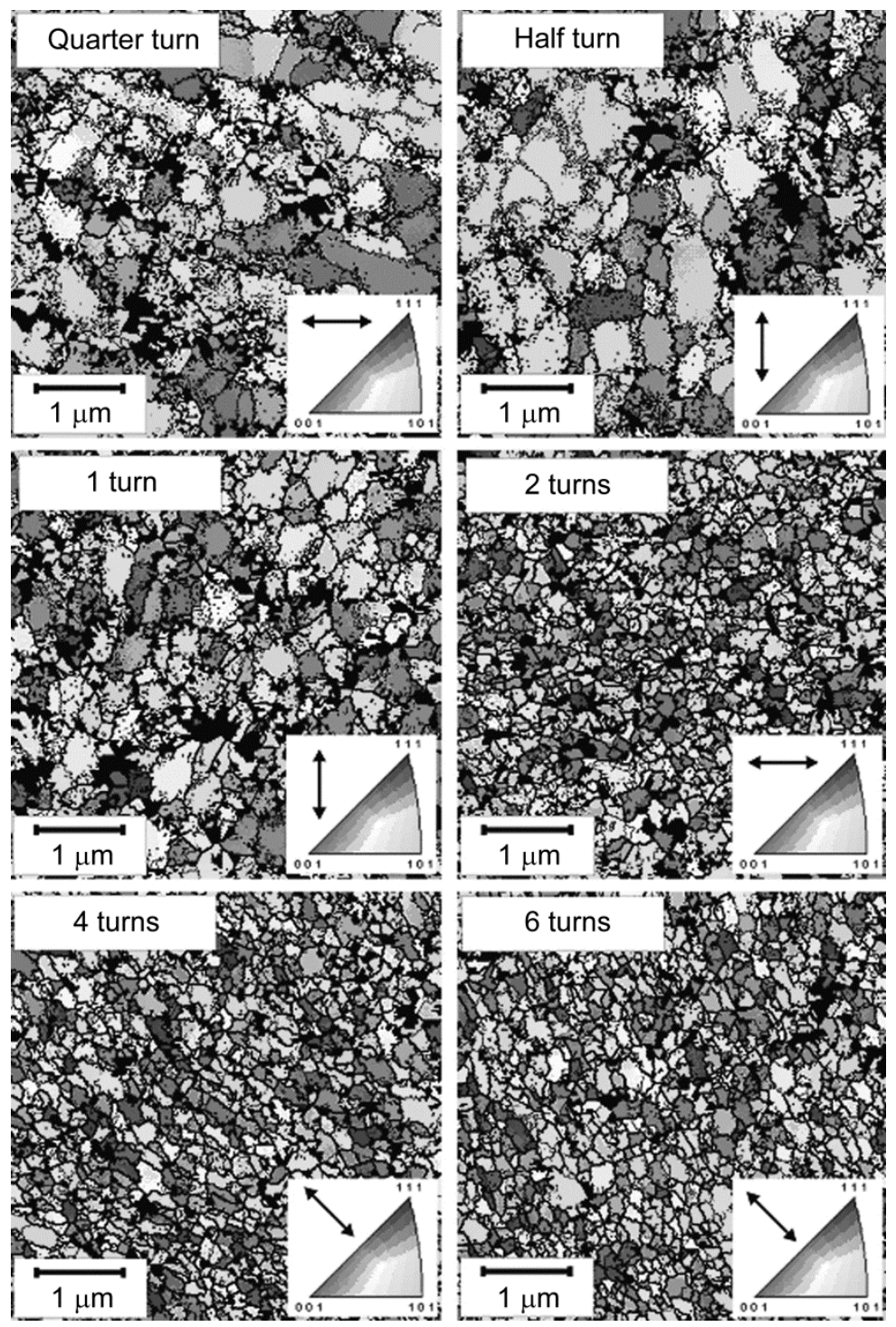

Fig. 2. OIM maps of $\mathrm{Fe}-3 \% \mathrm{Si}$ alloy microstructure after quasi-hydrostatic pressure. Colors indicate the crystallographic direction along the axis of torsion. Arrows indicate the shear direction.

size, that probably indicates to various structural mechanisms of grain and subgrain formation during megaplastic deformation.

A wide range of colors on the obtained OIM maps of crystal lattice orientations along the axis of torsion in the Bridgman anvil indicates to a relatively weak crystallographic texture. Uniaxial deformation at low temperatures often leads to the formation of a strong metallographic texture [10]. In our case, changes in the microstructure include the formation of almost equiaxed grains at all the deformation stages. The shear direction in Fig. 2 is detected by an insignificant grain elongation in the direction of plastic flow.

Attention should be drawn to Fig. 4 which plots the evolution of the grain size distribution during megaplastic deformation. After relatively small number of turns, the grain size distribution has a wide peak with a well-defined decay extending far towards coarser grains. With increasing number of turns, the peak of the grain size distribution narrows, and its maximum rises. The UFG or nanocrystalline structure formed after many turns is described by the symmetrical grain size distribution in relation to rather a sharp peak. Similar changes are observed in the subgrain size 


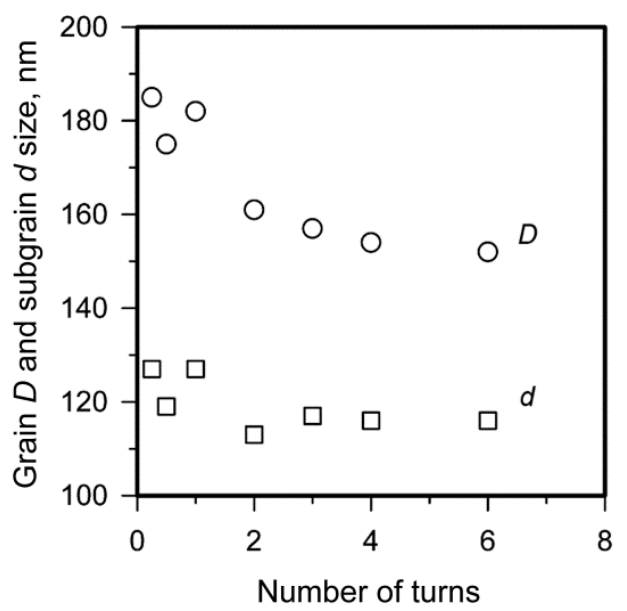

Fig. 3. Dependence of $D$ and $d$ grain and subgrain sizes in $\mathrm{Fe}-3 \% \mathrm{Si}$ alloy on quasi-hydrostatic pressure.

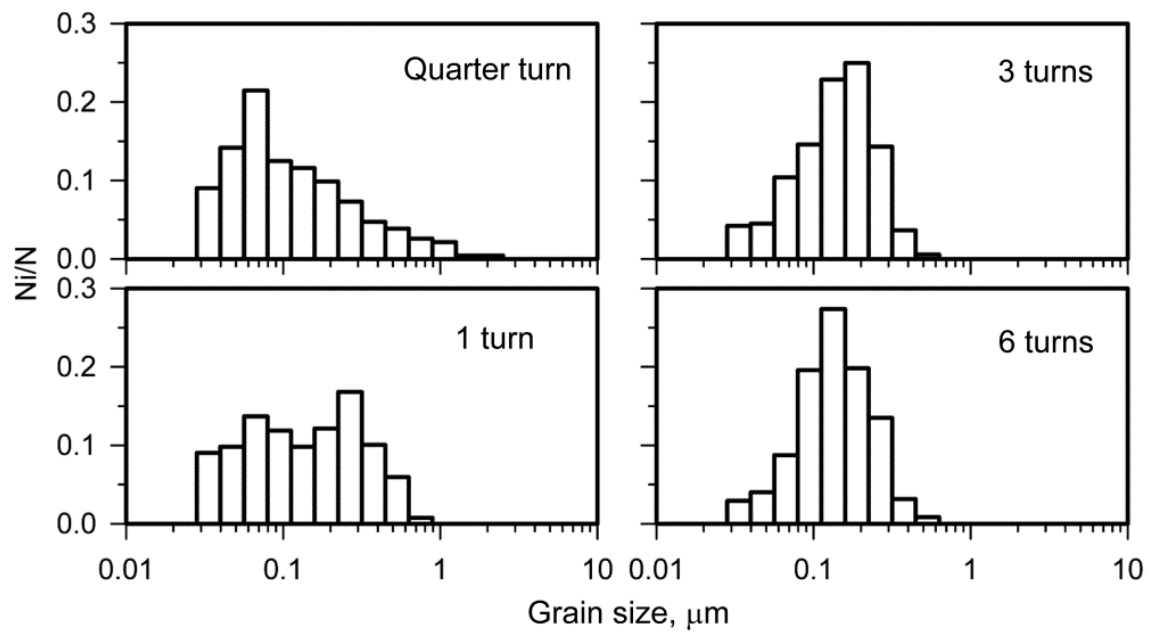

Fig. 4. Grain size distribution in Fe-3\% Si alloy after quasi-hydrostatic pressure.

distribution, which is shown in Fig. 5. A wide, asymmetrical peak appearing after a small number of turns becomes narrower and symmetrical when this number increases. This change in the grain and subgrain size distribution denotes, probably, a specific mechanism of the UFG and nanocrystalline structure formation, i.e. non-uniform formation of new, small grains. New grains occur mainly in places with high distortion gradient of the crystal lattice similar to the continuous dynamic recrystallization process suggested in [11] for the understanding of structural modifications due to megaplastic deformation at low temperatures.

A continuous formation of fine grains resulting from the substructure evolution during plastic deformation is indirectly supported by specific modifications of the grain-boundary misorientation distributions. This is illustrated in Fig. 6. After a small number of turns, we observe a well-defined, low-angle misorientations peak of less than 5 degrees. The amount of high-angle grain boundaries is low and does not depend on the grain-boundary misorientation angle. The total amount of high-angle grain boundaries does not exceed $20 \%$. With increasing deformation, the amount of highangle grain boundaries also increases. Among them one can distinguish grain-boundary misorientation of 40 degrees, whereas that of 20 degrees is described by a low specific value. The high-angle grain-boundary misorientation 


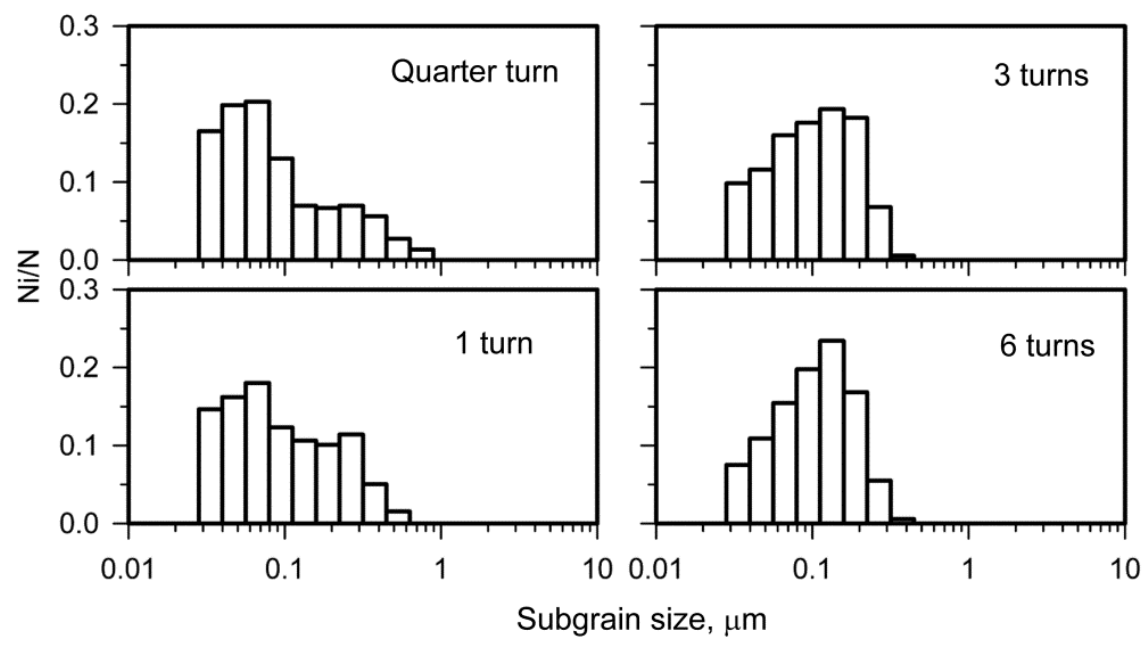

Fig. 5. Subgrain size distribution in $\mathrm{Fe}-3 \% \mathrm{Si}$ alloy after quasi-hydrostatic pressure.

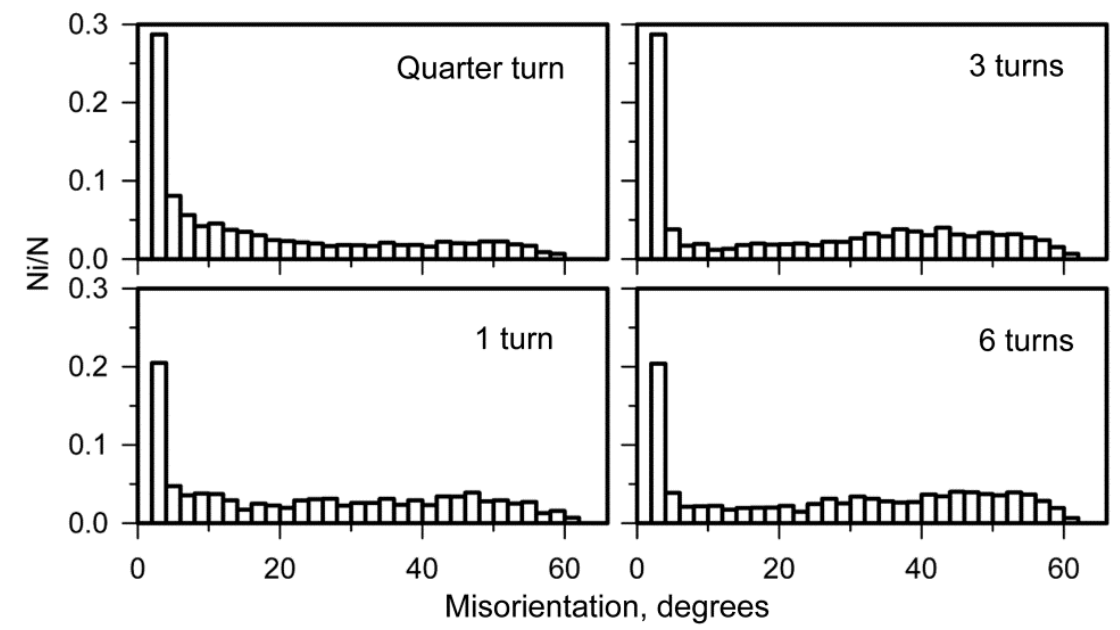

Fig. 6. Grain-boundary misorientation distribution in $\mathrm{Fe}-3 \% \mathrm{Si}$ alloy after quasi-hydrostatic pressure.

distribution, when the boundary value increases almost linearly with a misorientation angle increase up to 45 degrees followed by its drop to zero at a misorientation angle increase up to 62.8 degrees, is characteristic to a random misorientation distributions of cubic crystals [12]. Moreover, such a domination of highly mobile grain boundaries with a 40 degree misorientation angle absolutely indicates to an important role of the dynamic recrystallization in the fragmentation process during megaplastic deformation [13].

The increase in the high-angle grain boundaries during deformation correlates with the growth in the average grain-boundary misorientation angle, which is depicted in Fig. 7. Both of these parameters monotonely grow with increasing deformation. Such changes in grain-boundary misorientations are described in [11] during the continuous dynamic rectystallization. Deformation processing at $n<4$ is characterized by a comparatively high rate of change in grain-boundary parameters, whereas the subsequent increase in the number of turns up to 6 is accompanied by an insignificant change in the amount of high-angle grain boundaries and the average grain-boundary misorientation angle. It is interesting to note that relatively small deformations cause the strongest changes in physical properties of 


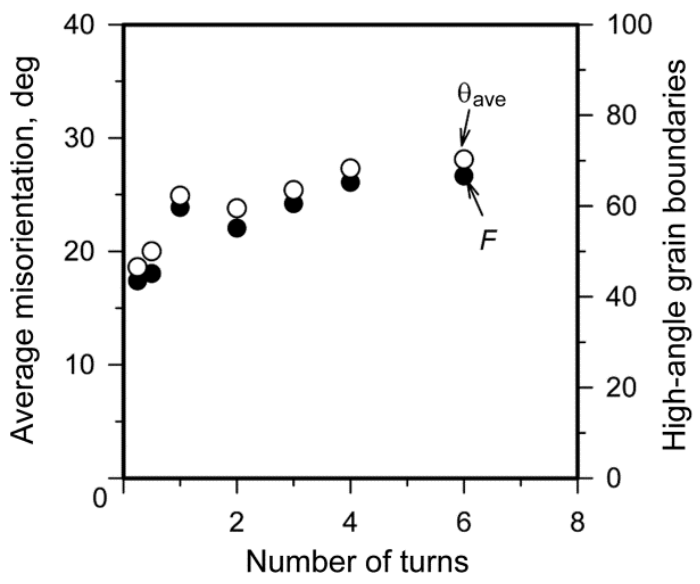

Fig. 7. Dependence of high-angle grain boundaries $(F)$ and average grain-boundary misorientation angle $\left(\theta_{\text {ave }}\right)$ on quasi-hydrostatic pressure in $\mathrm{Fe}-3 \% \mathrm{Si}$ alloy.

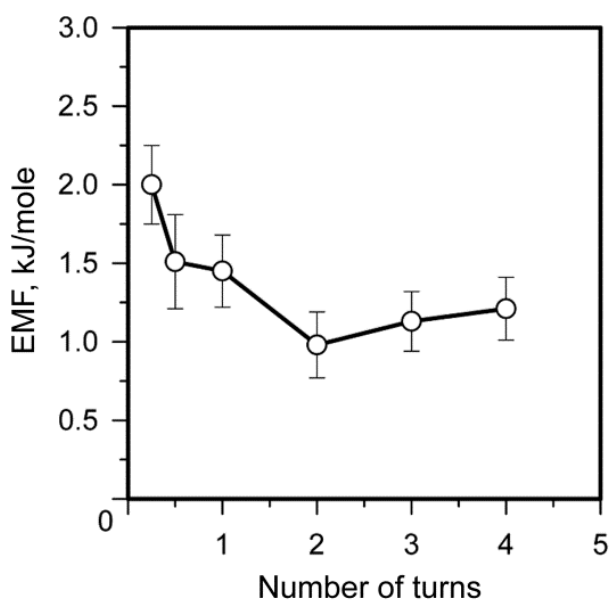

Fig. 8

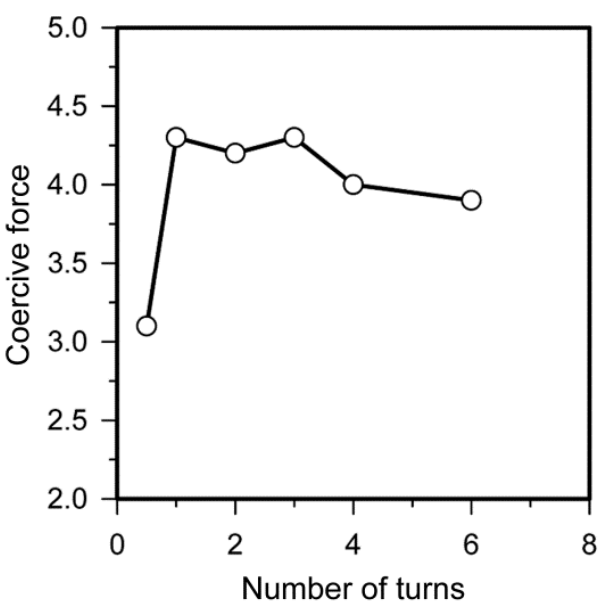

Fig. 9

Fig. 8. Dependence between quasi-hydrostatic pressure and electromotive force in $\mathrm{Fe}-3 \% \mathrm{Si}$ alloy.

Fig. 9. Dependence between quasi-hydrostatic pressure and coercive force in $\mathrm{Fe}-3 \% \mathrm{Si}$ alloy.

silicon iron subjected to quasi-hydrostatic pressure in the Bridgman anvil (Figs 8 and 9). The electromotive force describing the system relaxation reaches its minimum, while the coercive force reaches its maximum after 2 turns and a half turn, respectively. Thus, the stability of the microstructure parameters and physical properties in the large deformations range clearly demonstrates the presence of stabilized plastic deformation of silicon iron even in cold deformation conditions at room temperature. In this case, the steel microstructure and properties are defined by the dynamic equilibrium of such processes as cold hardening, fragmentation, recovery and recrystallization and can be represented by a continuous dynamic recrystallization $[11,14]$. This behavior of materials driven by megaplastic deformation is characteristic of the system working in terms of non-equilibrium thermodynamics [15]. 

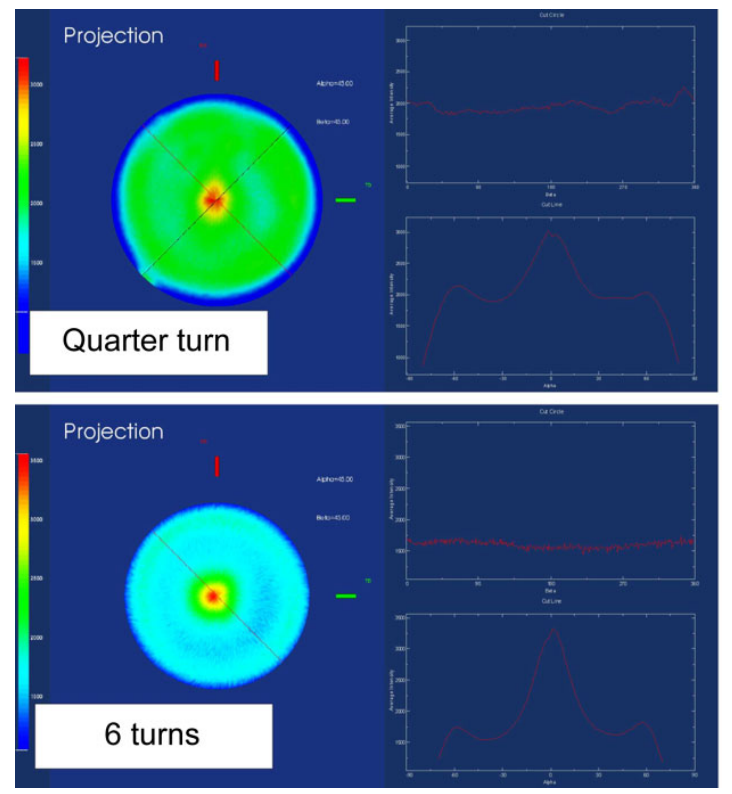

Fig. 10. (110)-oriented pole figures displaying the torsion plane of $\mathrm{Fe}-3 \% \mathrm{Si}$ alloy samples after quasi-hydrostatic pressure.

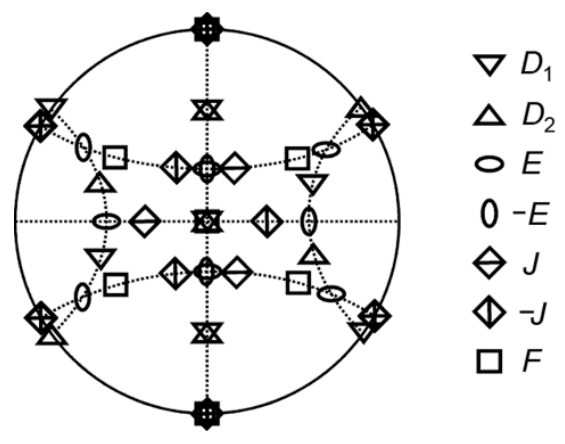

Fig. 11. Pole figure displaying ideal orientations of simple shear in BCC polycrystals.

Deformation of $\mathrm{Fe}-3 \% \mathrm{Si}$ alloy sample in the Bridgman anvil results in the formation of slip planes with a strong grain orientation parallel to the shear plane. After a quarter turn, the $<110>$-type orientation is observed along the axis of torsion, as displayed in Fig. 10 by pole figures. As can be seen, such a crystallographic texture is intensified with increasing deformation and the respective peak becomes sharper. The EBSD-assisted automatic analysis of the obtained data array on the individual grain orientation allows us to thoroughly study the evolution of the texture components during the alloy sample deformation. The ideal orientations of simple shear textures in BCC polycrystals is represented by the pole figure with (110) orientation in Fig. 11 and in Table 1 [16]. The texture with $<110>$ orientation along the axis of torsion formed after relatively small torsion deformation, includes such simple shear texture components as $\{110\}<111>,\{110\}<112>$ and $\{110\}<001>$, as mapped in Fig. 12. The intensity of $\{110\}<111>$ and $\{110\}<001>$ texture components is higher than that of $\{110\}<001>$. The increase in turns up to $n=1 / 2-1$ enhances the $\{110\}<001>$ component and increases the maximum pole density which achieves the highest value in this deformation range. Such an intensity ratio of the texture components is constant until large deformation values. After 6 turns, the intensity of the indicated components weakens with the formation of strong $\{110\}<112>$ and $\{112\}<111>$ texture components. 
TABLE 1. Ideal Orientations of Simple Shear in BCC Polycrystals

\begin{tabular}{|c|c|c|c|c|}
\hline \multirow{2}{*}{ Notation } & $\multirow{2}{*}{h k l}<\mathrm{uvw}>$ & \multicolumn{3}{|c|}{ Euler angles } \\
\hline & & $\varphi_{1}$ & $\phi$ & $\varphi_{2}$ \\
\hline$D_{1}$ & $\left(\begin{array}{lll}-1 & -1 & 2\end{array}\right)\left[\begin{array}{lll}1 & 1 & 1\end{array}\right]$ & $54.74 / 234.74$ & 45 & $0 / 90$ \\
\hline$D_{2}$ & $\left(\begin{array}{lll}1 & 1 & -2\end{array}\right)\left[\begin{array}{lll}1 & 1 & 1\end{array}\right]$ & $\begin{array}{c}125.26 \\
35.26 / 215.26\end{array}$ & $\begin{array}{l}45 \\
90\end{array}$ & $\begin{array}{c}0 / 90 \\
45\end{array}$ \\
\hline E & $\left(\begin{array}{lll}1 & 1 & 0\end{array}\right)\left[\begin{array}{lll}1 & -1 & 1\end{array}\right]$ & 90 & 35.26 & 45 \\
\hline$-E$ & $\left(\begin{array}{lll}-1 & -1 & 0\end{array}\right)\left[\begin{array}{lll}-1 & 1 & -1\end{array}\right]$ & 270 & 35.26 & 45 \\
\hline$J$ & $\left(\begin{array}{lll}1 & 1 & 0\end{array}\right)\left[\begin{array}{lll}1 & -1 & 2\end{array}\right]$ & $90 / 210$ & 54.74 & 45 \\
\hline$-J$ & $\left(\begin{array}{lll}-1 & -1 & 0\end{array}\right)\left[\begin{array}{lll}-1 & 1 & -2\end{array}\right]$ & $30 / 150 / 270$ & 54.74 & 45 \\
\hline$F$ & $\left\{\begin{array}{lll}1 & 1 & 0\end{array}\right\}<\begin{array}{lll}0 & 0 & 1>\end{array}$ & $\begin{array}{c}0 / 180 \\
90 / 270\end{array}$ & $\begin{array}{l}45 \\
90 \\
\end{array}$ & $\begin{array}{c}0 / 90 \\
45\end{array}$ \\
\hline
\end{tabular}

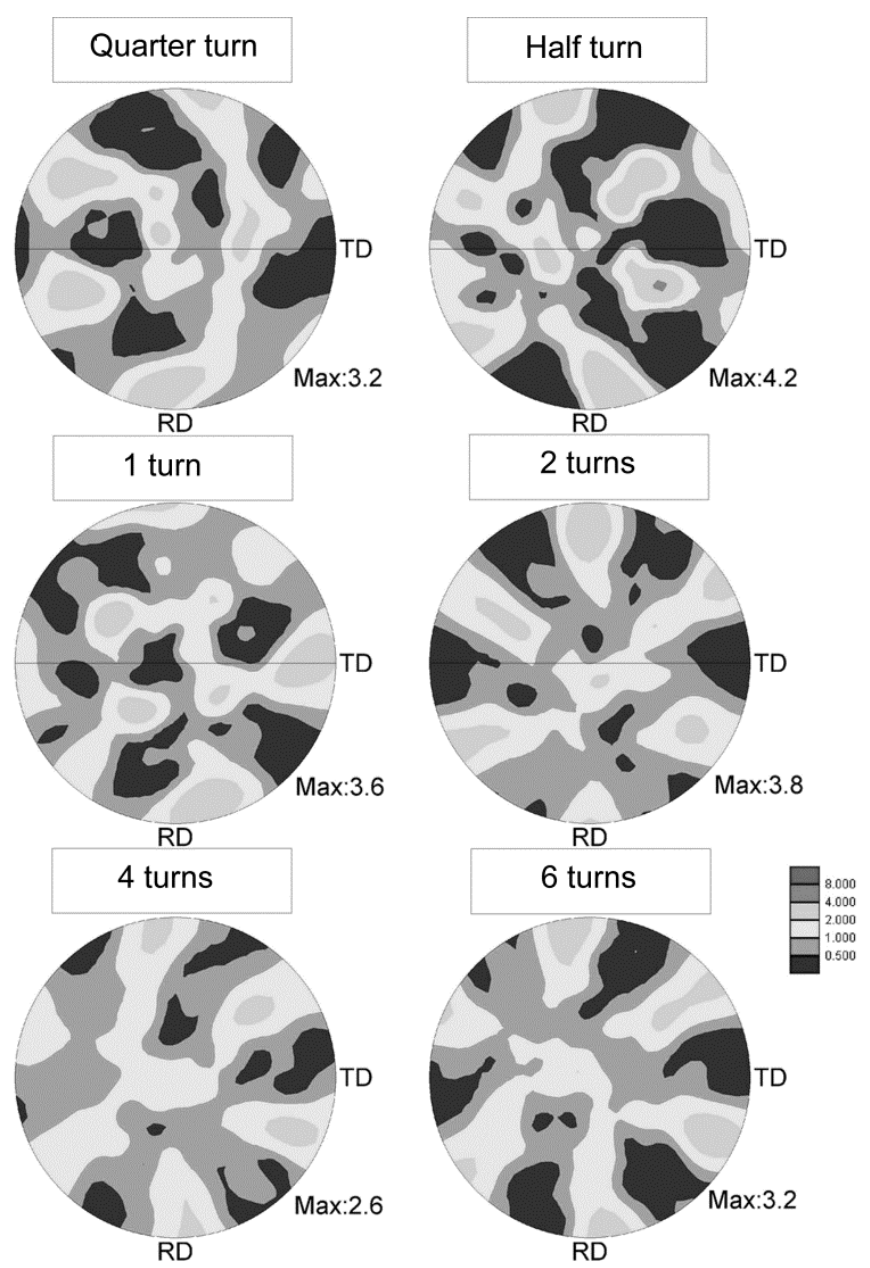

Fig. 12. (110)-oriented pole figures displaying the crystallographic texture of $\mathrm{Fe}-3 \% \mathrm{Si}$ alloy samples after quasi-hydrostatic pressure. TD - shear direction, $\mathrm{RD}-$ direction of axis of torsion. 

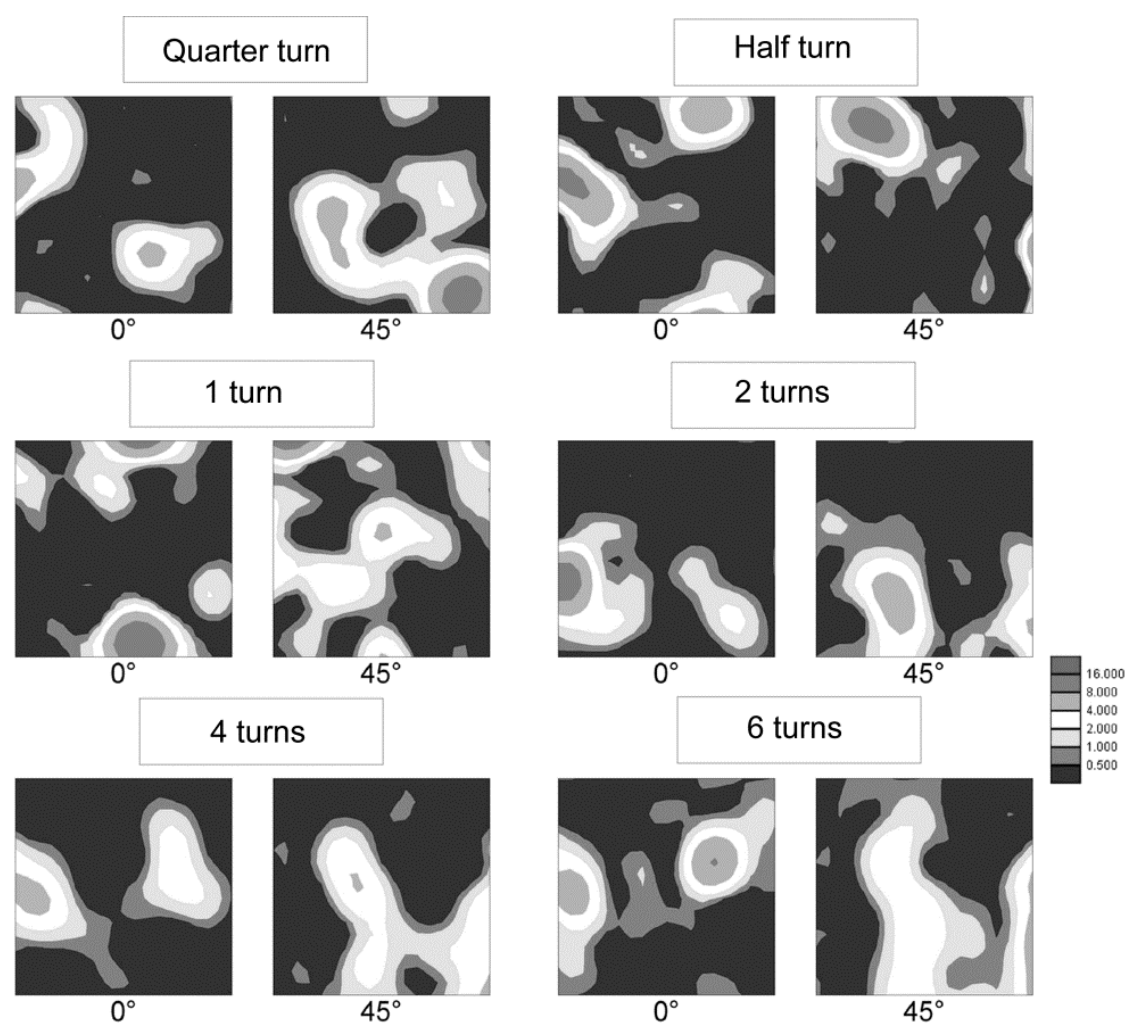

Fig. 13. Orientation distribution sections of functions in $\mathrm{Fe}-3 \% \mathrm{Si}$ alloy samples after quasi-hydrostatic pressure.
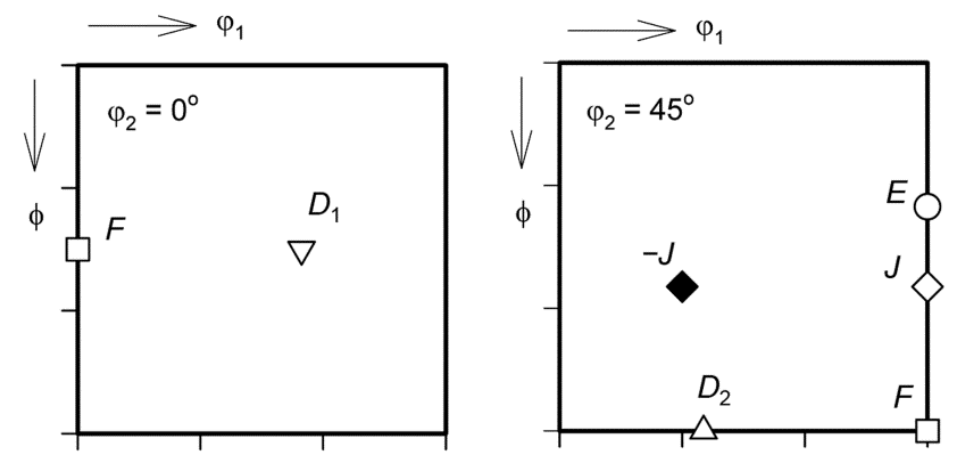

Fig. 14. Positions of ideal orientations of simple shear textures in BCC polycrystals.

Sections of functions of the orientation distribution obtained from the EBSD analysis of the XRD patterns are presented in Fig. 13 for $\varphi_{2}=0$ and $45^{\circ}$. Positions of typical ideal orientations of textures are given in Fig. 14. According to Fig. 13, after 2 turns the alloy sample texture includes all the components typical to simple shear. But after relatively small deformations $(n=1)$, the crosses correspond to the strong $\{100\}<011>$ texture component known as a rotated cube texture. The development of such a texture component is common to cold rolling textures for BCC metals and alloys [10]. It is obvious that prior to the deformation stabilization, the plastic flow of polyscrystals is accompanied by the grain fragmentation and rotations of separate microvolumes at a substructure level, which appear in conventional techniques of metal processing. On the other hand, rather large deformations usually result in the formation of a stable texture which does not change in further deformation. Nevertheless, this fact does not indicate to the stabilized stage of 
plastic flow during cold deformation of silicon iron, when its microstructure, texture and properties do not change significantly. These conditions can be achieved by using specific processing techniques, when the deformation system is subject to the laws non-equilibrium thermodynamics [15].

\section{CONCLUSIONS}

Deformation of $\mathrm{Fe}-3 \% \mathrm{Si}$ alloy due to torsion, under high quasi-hydrostatic pressure in the Bridgman anvil was accompanied by its significant hardening. The alloy sample microhardness increased from $2250 \mathrm{MPa}$ in the initial state up to $4320 \mathrm{MPa}$ after 6 turns of the movable anvil. At the same time, the most intense hardening was observed after 1 turn, while further deformation was accompanied by the gradual microhardness increase. The average grain size rapidly decreased down to $185 \mathrm{~nm}$ after a quarter turn and with increasing number of turns up to 6 it gradually decreased down to $150 \mathrm{~nm}$. The subgrain size weakly depended on the deformation degree. After a quarter turn, its size was $125 \mathrm{~nm}$, which decreased down to $115 \mathrm{~nm}$ after 6 turns. The texture development was characterized by the formation of the strong $\{001\}<110>$ texture component after relatively small deformations followed by the simple shear texture formation after 4 turns and over. Physical properties of the alloy changed rather strongly at $n \leq 1 / 2$ (coercive force) and $n$ $\leq 2$ (electromotive force). Further deformation did not affect the physical properties of the alloy. The stability of the alloy structure, texture and properties during large deformations indicated to a possible stabilization of plastic flow even at deformation temperatures below $0.2 \mathrm{~T}_{\mathrm{m}}$, which was associated with the evolution of the deformation system in accordance with the laws of non-equilibrium thermodynamics.

This work was carried out within the government contract N 2017/113 (2097) of the Ministry of Science and Higher Education of the Russian Federation. Experiments on sample deformation in the Bridgman anvil were supported by the Russian Science Foundation (Project No. 19-72-20066).

\section{REFERENCES}

1. U. Messerschmidt, Dislocation Dynamics During Plastic Deformation. Springer, Heidelberg (2010), 503 p.

2. J. P. Liu, E. Fullerton, O. Gutfleisch, and D. J. Sellmyer, Nanoscale Magnetic Materials and Applications. Springer, Heidelberg (2010), $450 \mathrm{p}$.

3. R. Z. Valiev, R. K. Islamgaliev, and I. V. Aleksandrov, Progr. Mater. Sci., 45, 103-189 (2000).

4. A. P. Zhilyaev and T. G. Langdon, Progr. Mater. Sci., 53, 893-979 (2008).

5. A. Belyakov and R. Kaibyshev, Nanostruct. Mater., 6, 893-896 (1995).

6. Glezer A. M., Kozlov E. V., et al., Plastic Deformation of Nanostructured Materials, Taylor \& Francis Group, Boca Raton (2017), 320 p.

7. F. J. Humphreys, J. Microsc., 195, 170-185 (1999).

8. Rigaku PDXL 2, Rigaku J., 28, No. 1, 29-30 (2012).

9. A. M. Glezer, R. N. Rostovtsev, A. A. Tomchuk, and I. V. Shchetinin, Bulletin of the Russian Academy of Sciences: Physics, 80, No. 80, 1027-1032 (2016).

10. L. A. I. Kestens and H. Pirgazi, J. Mater. Sci. Technol., 32, 1303-1315 (2016).

11. T. Sakai, A. Belyakov, R. Kaibyshev, et al., Progr. Mater. Sci., 60, 130-207 (2014).

12. J. K. Mackenzie, Biometrika, 45, 229-240 (1958).

13. A. M. Glezer and A. A. Tomchuk, Rev. Adv. Mater. Sci., 45, 9-15 (2016).

14. A. M. Glezer and R. V. Sundeev, Mater. Lett., 139, 455-457 (2015).

15. L. S. Metlov, Phys. Rev. Lett., 106, 165506-4 (2011).

16. S. Li, I. J. Beyerlein, and M. A.M. Bourke, Mater. Sci. Eng. A, A394, 66-77 (2005). 